Original Research Paper

\title{
Nonlinear Piecewise-Defined Difference Equations with Reciprocal Quadratic Terms
}

\author{
Ramadan Sabra and Saleem Shafiq Al-Ashhab \\ Department of Mathematics, Faculty of Science, Jazan University, Saudi Arabia \\ Department of Mathematics, Faculty of Science, Al-Albayt University, Jordan
}

\author{
Article history \\ Received: 21-09-2015 \\ Revised: 11-11-2015 \\ Accepted: 14-11-2015 \\ Corresponding Author: \\ Ramadan Sabra \\ Department of Mathematics, \\ Faculty of Science, Jazan \\ University, Saudi Arabia \\ E-mail: rmsabra@jazanu.edu.sa
}

\begin{abstract}
In this study we consider the several types of piece-wise defined sequences. We prove some results concerning the behavior of the sequence. In some cases we prove that the terms repeat themselves in certain patterns and we prove in other cases that the terms grow monotonically after few terms and approaches infinity.
\end{abstract}

Keywords: Difference Equations, Discrete Dynamical Systems, Bifurcation, Periodic Sequences

\section{Introduction}

In the theory of difference equations the existence of periodic solutions plays an important role. Applications of these classical existence theorems are well known now. Recently, these theorems have been generalized. For detail, see the research papers (Hou et al., 2012; Papaschinopoulos et al., 2007; Stevic, 2007; Zhang et al., 2000). In this study we present some definitions from discrete dynamical systems and introduce new some definitions and theories. One aspect of this study is to study the existence of fixed points and points having finite period of certain systems. Further, we find solutions for some difficult equations.

Han and Cheng (2012) considered the following nonlinear difference equation:

$$
x_{n}=a x_{n-2}+b f_{\lambda}\left(x_{n-1}\right)+c
$$

where, the function $f_{\lambda}$ is a piece-wise defined function as follows:

$$
\begin{aligned}
& f_{x}(x)=1 \text { for } x \in(0, \lambda) \\
& f_{x}(x)=0 \text { for } x \in(\lambda, \infty)
\end{aligned}
$$

They studied cases when the sequence is periodic or unbounded. This difference equation is equivalent to the system:

$$
u_{n}=y_{n-1} y_{n}=a u_{n-1}+b f_{\lambda}\left(y_{n-1}\right)+c
$$

Using the notation:

$$
p=\frac{c}{1-a}, q=\frac{b+c}{1-a}
$$

They found:

- If $0<\lambda<\mathrm{p}$, then all solutions of the system converge to the point ( $p ; p)$.

- If $\lambda=p$, then all solutions of the system such that $\mathrm{u}_{0}, \mathrm{y}_{0}$ $\in(p, \propto)$ converge to $(p ; p)$ and for the remaining start values converges to the limit 2 -cycle ( $p ; q),(q ; p)$

- If $\mathrm{p}<\lambda<\mathrm{q}$, then all solutions of the system converge to the limit 2-cycle (p; q), (q; p)

- If $\lambda=\mathrm{q}$, then all solutions of the system such that $\mathrm{u}_{0}$, $\mathrm{y}_{0} \in \Phi$ a specific subset of $\mathrm{R}^{2}$ converge to (q; $q$ ) and for the remaining start values converges to the limit 2-cycle (p; q), (q; p)

- If $\lambda>\mathrm{q}$, then all solutions of the system converge to $(\mathrm{q} ; \mathrm{q})$

Al-Ashhab and Guyker (2012) started in 2011 research on a new type of nonlinear difference equations. They considered a hand side of the difference equations, which consists of a piecewise defined function in two ways. This function is linear in the two ways. This made things easier to treat. Some results were proven, while other results are just conjectured. This is a new approach of dealing with difference equations, which posses periodic solutions. Qena et al. (2012) took further steps in the direction of considering nonlinear equations. They have a right hand side of the difference equations, which consists of a piece-wise defined function in two ways. But, this function is nonlinear in one of the two ways. The theory developed in (Qena et al., 2012) is more 
difficult to understand than in (Al-Ashhab and Guyker, 2012) and uses more complicated mathematical techniques. In (Qena et al., 2012; Sabra and Al-Ashhab, 2015a; 2015b) and (Al-Ashhab and Sabra, 2015) we find cases, in which unbounded solutions exists. The researchers did not explore all possible cases when the piece-wise defined function consists of linear branches. They left some room for research in this direction. Also, in the case that the function is nonlinear in one of the two branches we see only quadratic terms. Of course, quadratic equations are in a natural sense the next step when we want to have nonlinear terms and provide more room for proving theorems rather that cubic or higher order equations.

\section{General Sequences}

In this study we introduce the sequence defined as follows:

$$
L_{k}=\left\{\begin{array}{l}
h L_{k-1}^{\tau}, L_{k-1} \leq v, \text { for } k=1,2, \ldots \\
h L_{k-1}^{\theta}-j, L_{k-1}>v
\end{array}\right.
$$

The numbers:

$$
L_{0}, h, j, v, \tau, \theta
$$

Are determined according to certain specifications. This type of sequences was studied by the authors in other papers (Al-Ashhab and Sabra, 2015).

\section{Sequences with $\tau=-1$}

The number $\tau$ is set to minus one. We can see that in some cases the sequences is not well-defined. For example, if we set:

$$
h=2, v=1, j=4, L_{0}=1
$$

Then we obtain:

$$
L_{1}=\frac{h}{1}=2, L_{2}=2 * 2-4=0, L_{3}=\text { undefined }
$$

In case when the generated sequence continues welldefined to infinity we encounter different behavior types. In some cases we obtain simple periodic behavior as we shall show now:

Proposition 1: Let $h \geq 2$ and $n \geq 3$. If we choose:

$$
\begin{aligned}
& j=\frac{\left(h^{n}-1\right)(h-1)}{h^{n-1}-1}, \\
& 1<v<\frac{h^{2}-h^{n-1}+h^{n-2}-1}{h^{n-1}-1},
\end{aligned}
$$

Then we obtain the sequence:

$$
1, h, L_{2}, \ldots, L_{n}=1
$$

Proof: We note first that:

$\frac{h^{n}-h^{n-1}+h^{n-2}-1}{h^{n-1}-1} \leq h$

Since:

$$
\begin{aligned}
& h^{n}-h^{n-1}+h^{n-2}-1<h^{n}-h \Leftrightarrow \\
& h^{n-1}-h^{n-2}+1>h
\end{aligned}
$$

Which is true according to our choice of $h$ and $n$. Since the function:

$$
f(x)=h^{x-1}-h^{x} \text { for } x>1
$$

Is decreasing, we get:

$v<\frac{h^{2}-h^{n-1}+h^{n-2}-1}{h^{n-1}-1} \leq \frac{h^{2}-h^{m}+h^{m-1}-1}{h^{n-1}-1}$

for all $m=2, \ldots, n-1$

Now, we compute the terms of the sequence based on (3) and (4) as follows:

$$
\begin{aligned}
& L_{0}=1<v, L_{1}=h>v, L_{2}=h^{2}-j= \\
& h^{2}-\frac{\left(h^{n}-1\right)(h-1)}{h^{n-1}-1}=\frac{h^{n+1}-h^{2}-\left(h^{n}-1\right)(h-1)}{h^{n-1}-1}= \\
& \frac{h^{n}-h^{2}+h-1}{h^{n-1}-1}>v
\end{aligned}
$$

Similarly, we can show that:

$L_{m}=\frac{h^{n}-h^{m}+h^{m-1}-1}{h^{n-1}-1}>v$ for all $m=2, \ldots, n-1$

Thus, we obtain from (5):

$$
L_{n}=\frac{h^{n}-h^{n}+h^{n-1}-1}{h^{n-1}-1}=1 \bullet
$$

We consider a complementary case for $v$. Proposition 2: Let $h \geq 2$ and $n \geq 3$. If we choose:

$$
v>\max \left\{1, \frac{h^{2}-h^{n-1}+h^{n-2}-1}{h^{n-1}-1}\right\}
$$

Then we obtain for all values of $j$ the sequence:

$$
1, h, 1
$$


Proof: As in proof of Proposition 1 we know that:

$\frac{h^{n}-h^{n-1}+h^{n-2}-1}{h^{n-1}-1} \leq h$

Now, we compute the terms as follows:

$$
L_{0}=1<v, L_{1}=h<v, L_{2}=\frac{h}{h}=1 \bullet
$$

\section{Sequences with $\tau=-2$}

In this section we set the value of $\tau$ as minus two. We introduce the sequence:

$$
f_{i}=4^{i}-\frac{4^{i}-1}{3}, i=1,2,3
$$

Proposition 3: Let $h \geq 2, i \geq 1, \tau \geq-2$ and $\theta=1$. If we choose:

$$
L_{0}=h, j \in\left(0, h^{f_{i}+1}\right), h^{f_{i}} \leq v<h^{f_{i}+1}
$$

Then we get an unbounded sequence.

Proof: We note first that:

$$
\begin{gathered}
f_{1}=3, L_{0}=h<h^{3} \leq v, L_{1}=\frac{h}{h^{2}}=\frac{1}{h} \\
<h \leq v, L_{2}=h^{3}=h^{f_{1}} \leq v L_{3}=\frac{1}{h^{2 f_{1}-1}}<h \leq v \\
L_{4}=h h^{4 f_{1}-2}=h^{f_{2}}
\end{gathered}
$$

We continue in this manner till we reach :

$$
L_{2 i+2}=h^{f_{i+1}}>v, L_{2 i+3}=h h^{f_{i+1}}-j \geq 2 h^{f_{i+1}}-j>h^{f_{i+1}}>v
$$

We denote by:

$$
D=h^{f_{i+1}}-j>0
$$

Now we prove by induction that:

$$
L_{2 i+2+k} \geq 2^{k} D+j>v, k=1,2
$$

We take as basis step:

$$
L_{2 i+2+1}=L_{2 i+3} \geq 2 D+j=2 h^{f_{i+1}}-j
$$

We suppose now that for some k we have:

$$
L_{2 i+2+k} \geq 2^{k} D+j>v
$$

Hence:

$$
L_{2 i+2+k+1}=h L_{2 i+2+k}-j \geq 2\left(2^{k} D+j\right)-j=2^{k+1} D+j
$$

Since $2^{\mathrm{k}} \mathrm{D}<2^{\mathrm{k}+1} \mathrm{D}$ we obtain:

$$
L_{2 i+2+k+1}>2^{k+1} D+j=v
$$

Equation (9) represents the induction step.

Proposition 4: Let $h \geq 2, i \geq 1, \tau=-2$ and $\theta=1$. If we choose:

$$
L_{0}=h, j=h^{f_{i+1}+1}-a, a \in\left\{1, h, h^{f_{1}}, \ldots ., h^{f_{i}}\right\}
$$

Then we obtain a periodic sequence.

Proof: We start like this:

$$
\begin{aligned}
& L_{0}=h, L_{1}=\frac{1}{h}, L_{2}=h^{f_{1}}, \ldots, L_{2 i}=h^{f_{i}}, \\
& L_{2 i+1}=\frac{h}{h^{2 f_{i}}}, L_{2 i+2}=h^{f_{i+1}}>v L_{2 i+3}=h h^{f_{i+1}}-j=a
\end{aligned}
$$

But $a$ is an element of the set $\left\{\mathrm{L}_{0}, \mathrm{~L}_{2}, \mathrm{~L}_{4}, \ldots, \mathrm{L}_{2 \mathrm{i}}\right\}$. Hence we obtain a periodic sequence.

Proposition 5: Let $h \geq 2, i \geq 2, \tau=-2$ and $\theta=1$. If we choose:

$$
L_{0}=h, j>h^{2 f_{i+1}}, h^{f_{i}} \leq v<h^{f_{i+1}}
$$

Then we get an unbounded sequence.

Proof: As before we reach:

$$
L_{2 i+3}=h^{f_{i+1}+1}-j<h^{f_{i+1}+1}-h^{2 f_{i+1}}<0<v
$$

Now we use the notation:

$$
x=h^{f_{i+1}}>h^{3}, \quad h \geq 2
$$

This allows us to write the following expression:

$$
\begin{aligned}
& L_{2 i+3}<h x-x^{2}<0 \\
& 0<L_{2 i+4}<\frac{h}{x^{2}(x-h)^{2}} \leq \frac{h}{h^{6}\left(h^{3}-h\right)} \leq 1<v \\
& L_{2 i+5}=\frac{h L_{2 i+3}^{4}}{h^{2}}=\frac{\left(h^{f_{i+1}+1}-j\right)^{4}}{h} \geq \frac{\left(j-h^{f_{i+1}+1}\right)^{4}}{h}
\end{aligned}
$$

According to our assumptions:

$$
h^{f_{i+1}+1}<h \sqrt{j}, j>h^{6}, h \geq 2
$$

Hence: 


$$
L_{2 i+5} \geq \frac{(j-h \sqrt{j})^{4}}{h}>j
$$

Since:

$$
\begin{aligned}
& j^{4}-4 h j^{3.5}+6 h^{2} j^{3}-4 h^{3} j^{2.5}+h^{4} j^{2}>j^{4}-h^{3} j^{3.5} \\
& +6 h^{2} j^{3}-h^{5} j^{2.5}+h^{4} j^{2}>h^{4} j^{2}>h j
\end{aligned}
$$

Since $\mathrm{j}>\mathrm{v}$ we conclude that $\mathrm{L}_{2 \mathrm{i}+6}=\mathrm{hL}_{2 \mathrm{i}+5}-\mathrm{j} \geq 2_{\mathrm{L} 2 \mathrm{i}+5}$ $-\mathrm{j}>\mathrm{L}_{2 \mathrm{i}+5}$.

We use the notation $\mathrm{D}=\mathrm{L}_{2 \mathrm{i}+6}-\mathrm{L}_{2 \mathrm{i}+5}>0$. We prove by induction that:

$$
L_{2 i+k} \geq 2^{k-6} D+j>v
$$

for $\mathrm{k}=6,7, \ldots$ But:

As basis step we take $k=6$. This yields $\mathrm{L}_{2 \mathrm{i}+6} \geq 2 \mathrm{D}+\mathrm{j}$.

$$
L_{2 i+6}=L_{2 i+5}+D>j+D>v
$$

We turn attention now to induction step: Suppose that for some $\mathrm{k} \geq 6$ the following relation holds:

$$
L_{2 i+k} \geq 2^{k-6} D+j>v
$$

Hence, according to definition:

$$
\begin{aligned}
& L_{2 i+k+1}=h L_{2 i+k}-j \geq 2 L_{2 i+k}-j \geq 2 \\
& \left(2^{k-6} D+j\right)-j=2^{k+1-6} D+j>v
\end{aligned}
$$

From (1) we deduce that the limit of $L_{n}$ is infinite.

In some cases there will be periodic behavior of the sequence. For example, the setting:

$$
h=2 j=2^{12}-2=4094 v \in[8,2047)
$$

Generates the sequence:

$$
2, \frac{1}{2}, 8, \frac{1}{32}, 2048
$$

We can prove the following.

Proposition 6: Let $h \geq 2$ and $\theta \geq 1$. If we choose:

$$
h \leq v<h^{3}, j=\frac{L_{0}^{4 \theta}}{h^{\theta-1}} \pm L_{0}, L_{0} \in[h, v]
$$

Then we get the sequence:.

$$
L_{0}, L_{1}, L_{2}, L_{3}, L_{1}
$$

Proof: We note that:

$$
j=\frac{L_{0} L_{0}^{3 \theta}}{h^{\theta-1}} \pm L_{0} \geq h L_{0}^{3 \theta} \pm L_{0} \geq C
$$

Since:

$L_{0} \leq v$

We obtain:

$$
\begin{aligned}
& L_{1}=\frac{h}{L_{0}^{2}} \leq \frac{1}{h}<h \leq v, L_{2}=\frac{h}{L_{1}^{2}}=\frac{L_{0}^{4}}{h} \geq \frac{h^{4}}{h}=h^{3}>v, \\
& L_{3}=h L_{2}^{\theta}-j=h\left(\frac{L_{0}^{4}}{h}\right)^{\theta}-j=\frac{L_{0}^{4 \theta}}{h^{\theta-1}}-j= \pm L_{0}^{h}, L_{4}=\frac{h}{L_{0}^{2}}=L_{1}
\end{aligned}
$$

\section{Conclusion}

We developed theory for the new introduced concept of nonlinear piecewise-defined sequences, which are different than in the references. We determined some conditions under which the sequences have some periodic behavior. But, the sequence becomes sometimes unbounded. There are still open problems to solve in this direction. Actually, our observations using the computer lead us to the guess that the bounded sequences are such these sequences, which we considered here.

In this paper we pave a way how to start a bifurcation analysis with respect to some parameters like $j$ or $v$ since the sequences changes behavior for different settings. For example, when we consider:

$$
L_{0}=h, j \in\left(0, h^{f_{i}+1}\right), h^{f_{i}} \leq v<h^{f_{i}+1}
$$

We obtain an unbounded sequence. While the choice:

$$
L_{0}=h, j=h^{f_{i+1}+1}-a, a \in\left\{1, h, h^{f_{1}}, \ldots, h^{f_{i}}\right\}
$$

Leads to a periodic sequence. But, we cannot till now state what happens for the other cases of $j$.

\section{Acknowledgement}

The authors would like to thank Academic Deanship in Jazan University for support.

\section{Author's Contributions}

All authors equally contributed in this work

\section{Ethics}

This article is original and contains unpublished material. The corresponding author confirms that all of the other authors have read and approved the manuscript and no ethical issues involved.

\section{References}

Al-Ashhab, S. and J. Guyker, 2012. Piecewise defined recursive sequences with application in matrix theory. J. Math. Computational Sci. 
Al-Ashhab, S. and R. Sabra, 2015. Nonlinear piecewisedefined and cubic difference equations. Proceedings of the 4th Annual International Conference on Computational Mathematics, Computational Geometry and Statistics (AIC' 15), Singapore.

Hou, C., L. Han and S.S. Cheng, 2012. Complete asymptotic and bifurcation analysis for a difference equation with piecewise constant control. Adv. Difference Equations.

Papaschinopoulos, G., C.J. Schinas and G. Stefanidou, 2007. On a $k$-order system of Lyness-type difference equations. Adv. Difference Equations. DOI: $10.1155 / 2007 / 31272$

Qena, M., S. Al-Ashhab and J. Guyker, 2012. Nonlinear piecewise defined difference equations. Int. Math. Forum, 7: 47-56.
Sabra, R. and S. Al-Ashhab, 2015a. Bifurcations of quadratic piece-wise defined recursive sequences. Int. Math. Forum, 10: 247-272.

Sabra, R. and S. Al-Ashhab, 2015b. Nonlinear piecewise-defined difference equations with reciprocal and cubic terms. GSTF J. Math. Statist. Operations Res., 3: 1-9.

Stevic, S., 2007. Asymptotics of some classes of higherorder difference equations. Discrete Dynamics Nature Society. DOI: $10.1155 / 2007 / 56813$

Zhang, L., G. Zhang and H. Liu, 2000. Periodicity and attractivity for a rational recursive sequence. J. Applied Math. Computations, 19: 191-201. DOI: $10.1007 /$ BF02935798 